\title{
Surface Potential Decay on Silicon Rubber Samples at Reduced Gas Pressure
}

\author{
Shahid Alam, Yuriy V. Serdyuk and Stanislaw M. Gubanski \\ High Voltage Engineering, Chalmers University of Technology, \\ 41296 Gothenburg, Sweden
}

\begin{abstract}
Accumulation of interfacial charges is an inherent feature of HVDC insulation based on solid and gaseous media. The collected surface charges can alter the geometrical electric field leading to undesirable phenomena such as partial discharges and even unexpected flashovers.

In the present paper, surface potential decay on silicone rubber samples is analyzed at reduced pressures of ambient air that allows for elimination of surface charge neutralization by gas ions. Thus, influences imposed by bulk and surface conduction in the solid material are studied by means of computer simulations and experimental measurements. The results allow for identifying levels of bulk and surface conductivities above which the corresponding charge decay mechanism becomes dominant. It is shown that with a negligible space charge effect and significant surface leakage, there exists a notable spread of charge along gas-solid interface yielding visible crossover phenomenon in charge decay characteristics. It is also demonstrated that the effect of space charge in the material bulk on surface potential decay can only be significant within layers of material finer than $\sim 100 \mu \mathrm{m}$.
\end{abstract}

\section{Introduction}

High voltage polymeric insulators usually operate under high electric stresses which may initiate electrical discharges in surrounding air leading to generation of charged species (ions) and their deposition on gas-solid interfaces. The accumulated surface charges can become strong and alter field distribution around the insulator that, in turn, affects insulator performance [1, 2]. For proper design of the insulation, it is important to understand processes associated with charge dynamics on polymeric insulator surfaces [2, 3]. In this respect, measurements of potential decay on corona charged samples can be used to characterize insulating materials and to provide information about fundamental mechanisms of charge transport [4].

Surface potential/charge decay on insulating polymers has been studied extensively during last decades and various models and hypothesis have been proposed to describe surface charge dynamics [2, $4-6]$. It is commonly accepted that the amount of charges on a polymeric surface can diminish due to several processes, namely, bulk and surface conduction in the solid and due to neutralization by ions present in gas phase [7]. Under normal conditions, all these mechanisms act simultaneously and it is impossible to evaluate contributions of processes in each material (solid and gas) to the total effect that is highly desirable for understanding of the phenomenon. In some situations, the intensity of neutralization of surface charges ions can be reduced by means of using a specific operating mode of the measuring sensor placed in the vicinity of the analyzed surface [2]. This, however, introduces a local disturbance in the charge decay process and can't prevent gas neutralization over the entire charged object. In the present study, the influence of the gas phase is eliminated by conducting surface potential measurements at reduced gas pressure that provides a low number of ions in the gas volume. Such approach allows for analyzing solely the role of solid material on surface charge dynamics. In the paper, the results of the measurements of surface potentials on charged silicon rubber samples are presented. A model of the decay process is introduced and the output from the performed simulations is compared with the experimentally obtained data. Results of a parametric study are presented to demonstrate the influences of the material properties (surface and bulk conductivities) and possible space charges in the material on surface potential profiles and decay characteristics.

\section{Experimental setup and procedure}

The experimental setup is built based on a gas pressure chamber (volume $\sim 1 \mathrm{~m}^{3}$ ) allowing for carrying out the measurements at low gas pressures. Inside the chamber, a linear positioning system with a movable table carrying a material sample is installed and it is connected to an external controller. The charging arrangement includes a corona needle, which is used to deposit charges onto a flat sample of polymeric material. The needle is mounted on a wooden arm and it is connected to an external HVDC generator through a high-voltage bushing of the chamber. The surface potential measuring set-up utilizes Kelvin-type vibrating probe (Trek 3455ET) installed on the same arm and connected to an electrostatic voltmeter (Trek 341B, \pm 20 $\mathrm{kV})$. The connection of both the positioning system and electrostatic probe to the external devices are realized via dedicated bushings, which are properly insulated from the grounded shell to carry out the signals between the external and internal circuits. A digital manometer with a precision of $0.1 \%$ is mounted through a valve on the wall of the chamber to monitor the pressure during the experiment. A vacuum pump is used to reduce the pressure inside the test vessel. 
Surface potential measurements were carried out on samples $100 \times 100 \times 2 \mathrm{~mm}^{3}$ of non-post cured silicone rubber placed on the movable table inside the test vessel. In the experiments, the surface of the material was first charged by applying DC voltage of $-12 \mathrm{kV}$ to the corona needle mounted above the sample (its tip was at $3 \mathrm{~mm}$ from the surface) during 2 minutes. Air pressure in the vessel was equal to the external atmospheric pressure and it was reduced down to $\sim 300 \pm 10$ mbar immediately after the charging was completed. During evacuation process, which normally took $\sim 3$ minutes, the table with the sample was brought to the position under the electrostatic probe and the measurement started upon the desired pressure was reached. To obtain a distribution of the potential on the surface, the sample was moved under the probe along the line starting from the edge to the center. Note that such a line scan reflected the potential distribution on the entire surface due to the symmetrical charging. The measurements of the surface potential distributions were repeated at different instants after charging and the obtained profiles were further utilized to deduce surface potential decay characteristics.

\section{Surface potential decay model}

As it is known, the relationship between the surface potential $\mathrm{V}_{\mathrm{s}}(\mathrm{V})$ and the surface charge density $\sigma\left(\mathrm{C} / \mathrm{m}^{2}\right)$ for flat material samples can be written as [8]

$\frac{\mathrm{d} \sigma(\mathrm{t})}{\mathrm{dt}}=\frac{\varepsilon_{r} \varepsilon_{0}}{\mathrm{~L}} \frac{\mathrm{d} V_{S}}{\mathrm{dt}}$

Here, $t$ stands for time, $\varepsilon_{0}$ is permittivity of vacuum, $\varepsilon_{r}$ is the dielectric constant of the material, and $L$ is the sample thickness. At the same time, the rate of change of the surface charge density can be linked to charge sources and sinks by utilizing current conservation conditions. Thus assuming the commonly accepted mechanisms mentioned above, one may write

$\frac{\mathrm{d} \sigma(\mathrm{t})}{\mathrm{dt}}=-j_{S}(\mathrm{t})-j_{b}(\mathrm{t})-j_{g}(\mathrm{t})$

where $j_{\mathrm{s}}$ is the current density due to surface conduction, $j_{b}$ is the current density due to charge leakage through the bulk, and $j_{g}$ is the current density caused by gas neutralization. The latter term can be ignored in further consideration due to the especially created conditions in the test vessel. The surface and bulk current densities in (2) can be expressed as

$J_{S}=\frac{d^{2} V_{S} / d s^{2}}{R_{S}}$

$J_{b}=\frac{V_{S}}{\rho \cdot \mathrm{L}}+\frac{9}{8} \cdot \varepsilon_{0} \varepsilon_{r} \cdot \mu \cdot \frac{V_{S}^{2}}{L^{3}}$

In (3), the derivative along the gas-solid interface $(s)$ is to be considered. The first term on the right hand side of (4) describes ohmic conduction while the second term represents the so-called space charge limited current SCLC ( $\mu$ stands for the mobility of charge carriers). The SCLC is known to be considerable under strong fields that may appear at high magnitudes of the surface potential. Inserting (3) and (4) into (2) and solving for the surface potential yields

$\frac{\partial V_{S}(t)}{\partial t}=-\frac{L \cdot d^{2} V_{S}(t) / d s^{2}}{\varepsilon_{0} \varepsilon_{r} \cdot R_{S}}-\frac{V_{s}(t)}{\varepsilon_{0} \cdot \varepsilon_{r} \cdot \rho}-\frac{9}{8} \cdot \mu \cdot \frac{V_{S}(t)^{2}}{L^{2}}(5)$

The initial conditions for (5) are taken from experimental data and it is used for obtaining potential distributions on the sample surface at longer instants.

\section{Computer implementation of the model}

Equation (5) incorporating surface and bulk conduction mechanisms was solved numerically using simulation tool Comsol Multiphysics, which is based on finite element method. The equation was implemented utilizing Partial differential equation (PDE) mode and using $1 \mathrm{D}$ axially symmetric model as measured surface potential distributions was found to be symmetrical about the mid position of the sample. In the selected 1D approach, the computational domain (line) represented the gas-solid interface and all the material parameters were taken as independent of the sample thickness. The PDE coefficients in (5) were calculated using characteristics of the material sample used in the experiments and shown in Table 1.

\section{Results and discussions}

The surface potential profiles obtained from both the experiments and simulations are presented in Figure1. Recall that the distributions shown correspond to a line from the edge of the sample (zero coordinate) to the location at which the corona needle was mounted during charging (approximately the middle of the sample) so the curves can be mirrored around this latter position. As it is seen, the potential profile after charging has a minimum at the center of the sample forming so-called saddle-shaped distribution. It is believed to be a consequence of back discharges compensating overcharging of the surface immediately after switching off the corona and grounding the corona electrode [2]. It can be observed that there is almost no spread of the potential along the surface with time that indicates the fact that surface conduction is not significant. The relatively large material thickness makes it hard to expect that the contribution of space charge in the

Table1. Material properties used in the simulation model.

\begin{tabular}{|c|c|c|}
\hline Parameters & Value & Reference \\
\hline$L, \mathrm{~mm}$ & 2 & sample \\
\hline $1 / R_{s}, \mathrm{Ssq}$ & $5 \cdot 10^{-19}$ & {$[2]$} \\
\hline $1 / \rho, \mathrm{S} / \mathrm{m}$ & $10^{-15}$ & {$[2]$} \\
\hline$\varepsilon_{\mathrm{r}}$ & 2.7 & {$[2]$} \\
\hline$\mu, \mathrm{m}^{2} \mathrm{~V}^{-1} \mathrm{~s}^{-1}$ & $10^{-14}$ & {$[9]$} \\
\hline
\end{tabular}




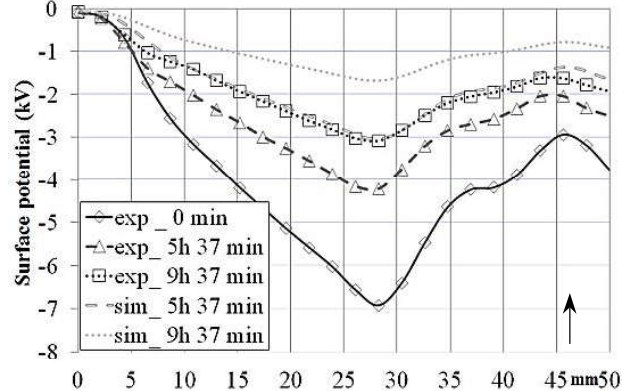

Fig 1. Surface potential profiles at different times after charging, the arrow indicates the location of the corona needle during charging.

material bulk can be significant. Therefore, one may suggest that the obtained time variations of the surface potential are affected mainly by charge leakage due to bulk conduction. This is also confirmed by the results of the simulations shown in Figure 1 (note that the experimental points for $9 \mathrm{~h} 37 \mathrm{~m}$ and the calculated results for $5 \mathrm{~h} 37 \mathrm{~m}$ are almost overlapping). For the material parameters used (Table 1), the second term in equation (5) was found to cause main influence on the solution. It is notable that the shapes of the calculated potential profiles at long times are similar to the measured ones (note that the experimental curve marked as " 0 min" was used as the initial condition). However, the quantitative differences between the experimental and simulation results increase with time that suggests that the actual value of the bulk conductivity doesn't remain constant with decreasing surface potential.

As it was discussed in [8], in case when bulk conduction is the dominant surface charge decay mechanism, the field dependent conductivity of silicon rubber can be related to the surface potential as

$\sigma\left(V_{S}\right)=\varepsilon \frac{1}{V_{S}(t)} \frac{d V_{s}(t)}{d t}$

and it can be fitted by e.g. Poole-Frenkel model. Within this approach, the conductivity is represented as a function of the square root of the surface potential

$\sigma\left(V_{S}\right)=\sigma_{0} e^{\beta \sqrt{V_{S}}}$

where $\sigma_{0}$ is the low field value, and $\beta$ is the factor defined as

$\beta=\frac{q}{k T} \sqrt{\frac{q}{\pi \varepsilon L}}$

Here, $q$ is the elementary charge; $k$ is Boltzmann's constant, and $T$ stands for temperature. The bulk conductivity of the studied silicone rubber deduced from the measured surface potential characteristics using the maximum magnitude of $V_{S}$ is shown in Figure 2 as a function of $\sqrt{ } V_{\mathrm{s}}$. As it is seen, two regions can be identified in the dependence: the conductivity is

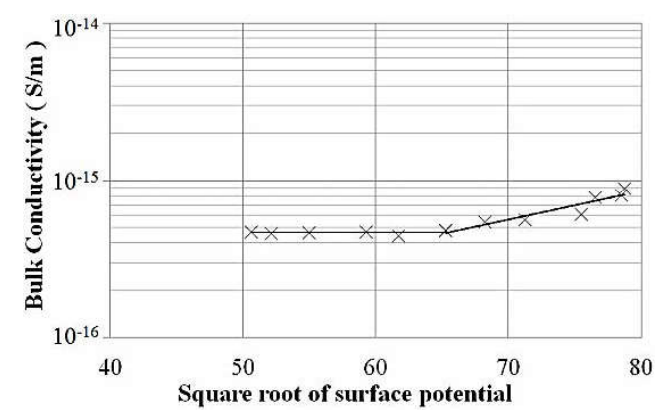

Fig 2. Field dependent bulk conductivity of the studied material. The lines are the fits of the experimental data.

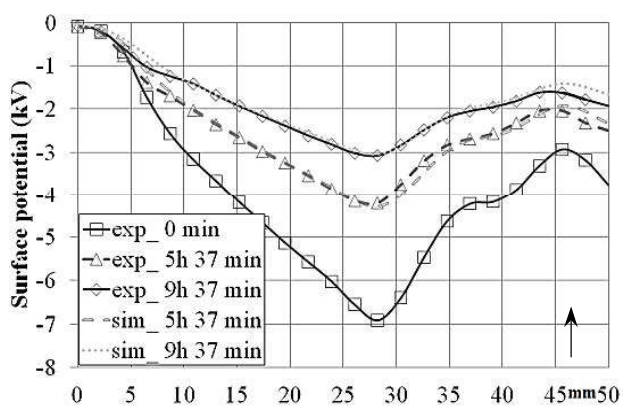

Fig 3. Surface potential profiles at different times after charging, the results of the simulations were obtained with the model accounting for the field dependent conductivity.

practically constant at $\sqrt{ } V_{S}$ below $\sim 65$ (that corresponds to $V_{S} \sim 4 \mathrm{kV}$ ) and it increases exponentially at higher values. Incorporating $\sigma\left(V_{S}\right)$ from Figure 2 into the model yielded perfect agreement with the experimental data shown in Figure 3. To get this fit, the conductivity value of $5 \cdot 10^{-16} \mathrm{~S} / \mathrm{m}$ was utilized, which is two times lower than that in Table 1 used for the calculations presented in Figure 1. The obtained agreement with the experimental data also reveals that bulk conduction is the dominant mechanism affecting charge decay under the conditions of the present study.

\section{Effect of material properties on surface potential decay}

The model equation (5) contains different terms that allows for evaluating their influences and relative importance in total potential decay. Thus, a parametric study was performed to analyze the effects of contributions of surface and bulk conductivities as well as the effect of SCLC and the results are shown below.

\subsection{Variation of surface conductivity}

The computed variations of surface potential profiles for two different values of surface conductivity are shown in Figure 4 for two different instants after charging. In the calculations, the bulk conductivity and the thickness of the material sample were kept as $10^{-15} \mathrm{~S} / \mathrm{m}$ and $2 \mathrm{~mm}$, respectively, and the curve marked as " 0 min" represents the initial conditions.From the simulations, it was found that the influence of surface conductivity was feasible when its magnitude exceeded $\sim 10^{-17} \mathrm{Ssq}$. As it 


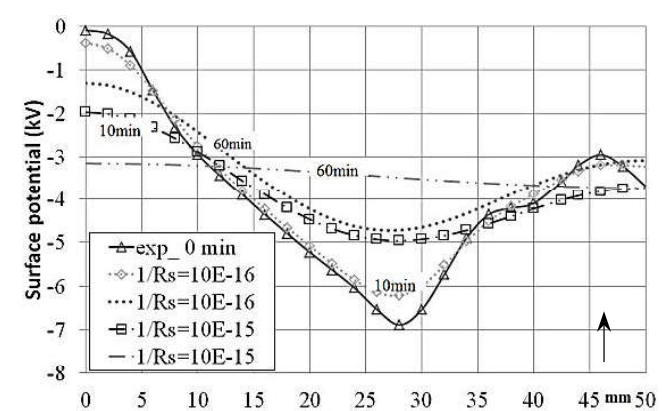

Fig 4. Surface potential profiles at different times computed with different values of surface conductivity $1 / R_{S}(\mathrm{Ssq})$.

is shown in the figure, the enhanced surface conduction intensifies charge spreading along the surface and may even result in a crossover in the surface potential profiles (observe curves for $1 / R_{S}=10^{-15} \mathrm{Ssq}$ ). It also leads to a faster potential decay as can be seen from the comparison of the data in Figures 1 and 4.

\subsection{Variation of bulk conductivity}

The surface potential distributions for two different values of the bulk conductivity at two different times are shown in Figure 5. In the calculations, the surface conductivity and the thickness of the material were kept as $10^{-17} \mathrm{Ssq}$ and $2 \mathrm{~mm}$, respectively.

As can be seen, as long as surface conduction is not significant, the crossover phenomena and charge spread along the surface is not prominent regardless of the value of the bulk conductivity. The increase in the bulk conductivity may only enhance the potential decay rate and it is hard to expect significant modifications in the shapes of surface potential profiles.

\subsection{Variation of the material thickness}

The calculations performed using the actual properties provided in Table 1 showed that decreasing the material sample thickness may lead to an increase in the space charge limited current (the last term in (5)). However, the effect may become significant only for thin films of the material with thicknesses below $\sim 100 \mu \mathrm{m}$ and it is negligible for the samples used in the present study.

\section{Conclusions}

The experimental study of surface potentials on corona charged silicone rubber samples has been performed at reduced pressure of surrounding air in order to analyze solely the effect of solid material properties on potential profiles and decay. The computer model accounting for charge leakage through material bulk and along gassolid interface has been developed.

Both the experiments and simulations demonstrated that bulk conduction became the dominant mechanism of surface potential decay if the volume conductivity of the material was above $\sim 10^{-16} \mathrm{~S} / \mathrm{m}$. The results of the modeling agreed well with the measured characteristics if field dependent conductivity of the material was taken into account. The performed parametric study showed

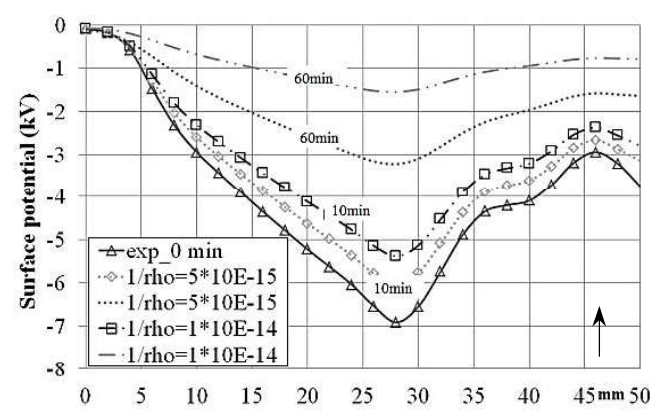

Fig 5. Surface potential profiles at different times computed with different values of bulk conductivity $1 / \rho(\mathrm{S} / \mathrm{m})$.

that surface conduction might influence the potential decay at magnitudes of surface conductivity higher than $\sim 10^{-17} \mathrm{Ssq}$. The effect of bulk space charges was found to be negligible for the conditions of the present study and its impact on the decay process might be expected at material sample thickness finer than $\sim 100 \mu \mathrm{m}$.

\section{References}

[1] S. Kumara, S. Alam, I. R. Houqe, Y. V. Serdyuk and S. M. Gubanski, "DC flashover characteristics of a cylindrical insulator model in presence of surface charges", IEEE Trans. Diel. Elec. Insul., Vol. 19, No. 3, pp. 1084-1090, June 2012.

[2] S. Kumara, "Electrical charges on polymeric insulator surfaces and their impact on withstand performance", $\mathrm{PhD}$ thesis, Chalmers University of Technology, Gothenburg, Sweden, 2012.

[3] F. Wang, Q. Zhang, Y. Qiu and E. Kuffel, "Insulator surface charge accumulation under DC voltage", Proc. IEEE Int. Symp. Elec. Insul., pp. 426 - 429, 2002.

[4] P. Molinie, "Measuring and modeling transient insulator response to charging: the contribution of surface potential studies", IEEE Trans. Diel. Elec. Insul., Vol. 12, No. 5, pp. 939-950, 2005.

[5] P. Molinie and P. Llovera, "Surface potential measurement: implementation and interpretation", IEEE Conf. Publ. No. 473, $8^{\text {th }}$ Int. Conf. Diel. Materials, Measurements and Applications, pp. 253-258, 2000.

[6] P. Molinie, "A review of mechanisms and models accounting for surface potential decay" IEEE Trans. Plasma Science, Vol. 40, No. 2, pp. 167-176, Dec. 2012. [7] J. Kindersberger and C. Liderle, "Surface charge decay on insulators in air and sulfurhexafluorid - part I: simulation", IEEE Trans. Diel. Elec. Insul., Vol. 15, No. 4, pp. 941-948, 2008.

[8] D. Min, M. Cho, A. R. Khan and S. Li, "Surface and volume charge transport properties of polyimide revealed by surface potential decay with genetic algorithm”, IEEE Trans. Diel. Elec. Insul., Vol. 19, No. 2, pp. 600-608, 2012.

[9] S. Le Roy, G. Teyssedre and C. Laurent, "Charge transport and dissipative processes in insulating polymers: experiments and model", IEEE Trans. Diel. Elec. Insul., Vol. 12, No. 4, pp. 644-654, 2005. 